\title{
COMPARING OF FAECAL CALPROTECTIN LEVELS IN PATIENTS WITH OSTEOARTHRITIS TAKING NSAID TREATMENT AND PATIENTS WITHOUT NSAIDS THERAPY
}

\author{
Olena Gubska1 \\ gubskao@gmail.com \\ Andrii Kuzminets ${ }^{1}$ \\ andrewkuzminets@gmail.com \\ Artem Panin ${ }^{1}$ \\ phoenix-art@ukr.net \\ ${ }^{1}$ Department of Therapy, Infectious Disease and Dermatology Postgraduate Education \\ Bogomolets National Medical University \\ 13 T. Shevchenko blvd., Kyiv, Ukraine, 01601
}

\begin{abstract}
Faecal calprotectin (FC) level can be increased in several conditions, which are characterised by neutrophilic inflammation. Some medications, particularly NSAIDs, can elevate its level as well. NSAIDs are taken by patients in many chronic conditions, including osteoarthritis (OA).

On the other hand, there is growing evidence that osteoarthritis is not only a degenerative disease, but it has a significant inflammatory component. The role of systemic inflammation is well-known in inflammatory joint diseases, but there is some evidence that it can play an essential role in the OA as well. It can suggest that in the OA, the inflammatory changes could be found in the different organs and systems.

The aim of this study was to investigate the FC level in patients with osteoarthritis depending on the NSAIDs intake and to compare it to the FC levels in healthy adults.

Materials and methods. In this small observational study, we evaluated the FC levels in patients suffering from OA (36 persons), divided them into two groups depending on their NSAIDs intake, and compared it to FC levels in healthy participants (12 persons). We compared the FC levels depending on the selectivity of the NSAIDs taken by our participants, as well.

Results. The median calprotectin level in our patients was 72.57 (IQR 20.55-221.57) mg/kg, $95 \%$ CI 26,18-109.01. OA patients had higher levels than the healthy group $(\mathrm{p}<0.001)$. OA patients who took NSAIDs had the highest FC levels -221.57 (IQR 91.56-448.61) - higher, than those who did not take it - 72.57 (IQR 35.26-164.79) ( $\mathrm{p}=0.03$ ) and than healthy participants, who has normal FC levels $(\mathrm{p}<0.001)$; the FC levels of patients who did not take NSAIDs also exceeds healthy subject's levels $(\mathrm{p}<0.001)$. The FC levels in the collective group have a sufficient positive correlation with the duration of NSAIDs intake, VAS score and strong correlation with Lequesne index values. We found that both NSAIDs groups have a significantly greater prevalence of elevated or high FC levels than the control group $(\mathrm{p}<0.001)$ and that NSAIDs patients significantly more often have high FC levels than those who do not take NSAIDs ( $\mathrm{p}=0.035$ ). When comparing FC levels in patients depending on the type of NSAIDs they take, we found that those who take non-selective NSAIDs has significantly higher FC levels than those who do not take NSAIDs - 264.1 (IQR 89.72-464.67) to 25.65 (IQR 19.5-75.33) ( $\mathrm{p}=0.0003)$. The FC levels of who take selective NSAIDs - 98.53 (91.56-105.5) - did not differ significantly to non-selective NSAIDs taker's group values and non-selective NSAIDs taker's values ( $p>0.05)$.

Conclusions. Patients who suffer from OA have higher FC levels than healthy individuals, and patients with OA who take NSAIDs regularly have higher FC levels than those who do not. The intake of non-selective NSAIDs is associated with higher FC levels, than the intake of high-selective NSAIDs. FC levels of those who take high-selective NSAIDs do not differ statistically from those who do not intake NSAIDs. Further research is needed in this area.
\end{abstract}

Keywords: NSAIDs, calprotectin, osteoarthritis, enteropathy, colopathy.

DOI: $10.21303 / 2504-5679.2020 .001193$

\section{Introduction}

NSAIDs are a group of medications that are commonly used by patients. It is well known for its harmful influence on the gastric mucosa, but it can affect any part of the gastrointestinal 
tract and can include a broad spectrum of gastrointestinal lesions [1, 2], including NSAID-induced enteropathy [3]. We described it in more detail in our last article [4]. These lesions are asymptomatic but can cause malabsorption, chronic protein, blood loss, and even, sometimes, intestinal obstruction [5, 6]. Commonly they can be found at videocapsule endoscopy, but several biomarkers can be used to find out if the NSAIDs-induced mucosal injury appeared. One of them is faecal calprotectin (FC).

Calprotectin is a cytosolic, calcium- and zinc-binding protein that can be found in neutrophils (predominantly), macrophages, and endothelial cells. The FC level relates quantitatively to acute intestinal inflammation [7]. The FC level can be elevated in several conditions, such as inflammatory bowel disease, coeliac disease, bacterial gastroenteritis, gastrointestinal tumours, diverticular disease, liver cirrhosis, or treatment with NSAIDs. It was shown that NSAIDs increase FC levels in $75 \%$ to $95 \%$ healthy individuals after two weeks of diclofenac plus omeprazole intake in therapeutic doses $[8,9]$. Other studies showed ibuprofen, celecoxib or naproxen, and omeprazole to increase FC levels. Many other investigations and reviews prove the NSAIDs influence on the FC level $[10,11]$. There are little evidence suggesting that acetaminophen does not alter FC levels [12].

One of the largest groups of patients taking NSAIDs are those suffering from osteoarthritis (OA). Many of them are aware of the negative influence of these medications on the stomach and trying to control it via controlling the symptoms of gastritis. The doctor can control it relatively easily, using upper endoscopy. However, there are no commonly used methods in clinical practice for controlling the lower intestinal side effects. Moreover, they are commonly asymptomatic $[4,13]$. The faecal calprotectin can be the suitable marker of NSAIDSinduced lesions of the lower GI tract in patients who do not have other conditions that lead to its increase in the stool. It is also recognised as a biomarker to distinguish organic intestinal diseases from functional ones $[14,15]$. On the other hand, in light of the new concept about low-grade inflammation subtype of OA, hypothetically, there can be inflammatory changes found in those patients, including high FC levels. Presently, there is a lack of knowledge on how often the FC level appears to be increased in patients with osteoarthritis taking NSAIDs and how high is its level in these patients.

The aim of this study was to investigate the FC level in patients with osteoarthritis who takes NSAIDS depending on the NSAIDs intake and to compare it to the FC levels in healthy adults.

\section{Materials and methods}

We conducted the study from April 2018 to April 2019 based on Department of Therapy, Infectious Disease and Dermatology Postgraduate Education of Bogomolets National Medical University (Kyiv). Stool samples were collected from consenting 36 patients who suffer from osteoarthritis (OA patients). Samples were selected and obtained from patients of Kyiv clinics and hospitals. The criteria of inclusion were instrumental-verified osteoarthritis of any joint (any X-ray stage). The exclusion criteria were: antibacterial medications, biologicals, sulphasalazine, misoprostol, bisphosphonates or laxatives at least for three months before study, verified or suspected structural diseases of the gastrointestinal tract such as inflammatory bowel disease, the malignancy of the gastrointestinal tract, intestinal infections, spondyloarthropathy, and seronegative arthritis, immunological disorders and an anamnesis of gastrointestinal surgery (except appendectomy or cholecystectomy). All the patient's pathologies were not known as a sources of high FC levels.

The study design was approved by the Commission on Bioethical Examination Questions and Ethics of Scientific Researches of Bogomolets National Medical University. The research included the Informed Concern Form, was not contained increased risk for study subjects and was conducted in conformity with the principles of the Helsinki Declaration of General Assembly of the World Medical Association (1964-2000) and the laws of Ukraine.

The mean age \pm standard deviation of patients was $59.63 \pm 14.11$ years, minimum and maximum age was 34 and 85 years, respectively. Between patients, there were fewer men (13-36.11\%) 
than women (23-63.89\%); the difference was statistically significant $(\mathrm{p}=0.033)$. All patients were divided into two groups: 1) patients taking NSAIDs regularly (NSAIDs group), 18 subjects, and 2) patients not taking NSAIDs (those who takes in less than once a week, or have taken them systematically, but cancelled more than two weeks before examination) (no NSAIDs group), 18 subjects (Table 1). Among patients 22 were normal to overweight $(61.1 \%)$, two patients $(5.6 \%)$ were underweight, and 12 persons (33.3\%) had obesity of different degrees; the difference is statistically significant $(\mathrm{p}<0.05)$. All patient's anamnesis of disease were detected for exclusion criteria. They were consulted and got instructions before passing stool tests. The samples were immediately collected and frozen $\left(-20^{\circ} \mathrm{C}\right)$. Patients from the no NSAIDs group did not take medications before the stool test (for headache, relief, instead).

Table 1

Characteristics of groups

\begin{tabular}{cccc}
\hline Group & $\begin{array}{c}\mathbf{n}: \\
\text { men; women }\end{array}$ & $\begin{array}{c}\text { \%: } \\
\text { men; women }\end{array}$ & $\begin{array}{c}\text { Average age } \pm \text { SD, } \\
\text { years }\end{array}$ \\
\hline NSAIDs $(\mathrm{n}=18)$ & $9 ; 9$ & $50 ; 50$ & $59.44 \pm 12.95$ years \\
No NSAIDs $(\mathrm{n}=18)$ & $4 ; 14$ & $22.2 ; 77.8$ & $59.82 \pm 15.64$ years
\end{tabular}

There was not found a significant difference in the number of patients, average age, gender composition, or body mass index (BMI) found between OA groups ( $p>0.05)$.

We recruited 12 healthy individuals (five men, seven women) as a control group.

Faecal calprotectin analysis and the stool sampling procedure

FC was determined by the RIDASCREEN® Calprotectin assay on Rayto RT-2100C reader. $50 \mathrm{mg} / \mathrm{kg}$ FC level was set as a positive value. The stool preparation was organised according to the recommendations of the kit manufacturer. The lower limit of detection was $19.5 \mathrm{mg} / \mathrm{kg}$, the higher limit $-800 \mathrm{mg} / \mathrm{kg}$.

All statistical analyses were conducted using EZR software v. 1.38 (Saitama Medical Center, Jichi Medical University, Saitama, Japan), which is a graphical user interface for R (The R Foundation for Statistical Computing, Vienna, Austria) [16]. We used the Kruskal-Wallis test adjusted by the Steel-Dwass amendment and Spearman's rank correlation test for non-parametric data and ANOVA analysis adjusted by the Tukey amendment for parametric tests. For qualitative data, we used Fisher's exact test adjusted by the Bonferroni amendment.

\section{Results}

The median FC level in our patients was 72.57 (IQR 20.55-221.57) mg/kg, 95 \% CI 26.18109.01. The results of FC levels in separate groups are shown in the Table 2 and Fig. 1.

Table 2

Medians of FC levels in groups

\begin{tabular}{ccc}
\hline Group & $\begin{array}{c}\text { Median (IQR), } \\
\mathbf{m g} / \mathbf{k g}\end{array}$ & $\begin{array}{c}\mathbf{9 5} \mathbf{\% C I}, \\
\mathbf{~ m g / k g}\end{array}$ \\
\hline NSAIDs $(\mathrm{n}=18)$ & $221.57(91.56-448.61)$ & $91.56-448.61$ \\
no NSAIDs $(\mathrm{n}=18)$ & $72.57(35.26-164.79)$ & $35.26-164.79$ \\
Healthy control $(\mathrm{n}=12)$ & $19,5(19.5-20.975)$ & $19.5-22.45$
\end{tabular}

The FC level was statistically different in these groups. Generally, OA patients showed significantly higher FC level than healthy subjects. The FC level was the highest in the group taking 
NSAIDs, the medium-high - in the group that was not taking NSAIDs and appeared to be normal in the control group.

We performed the comparison of visual analogue scale (VAS), Lequesne index, body mass index (BMI), and Bristol scale values, as potential confounders, between OA groups. None of them was statistically different $(\mathrm{p}>0.1)$.

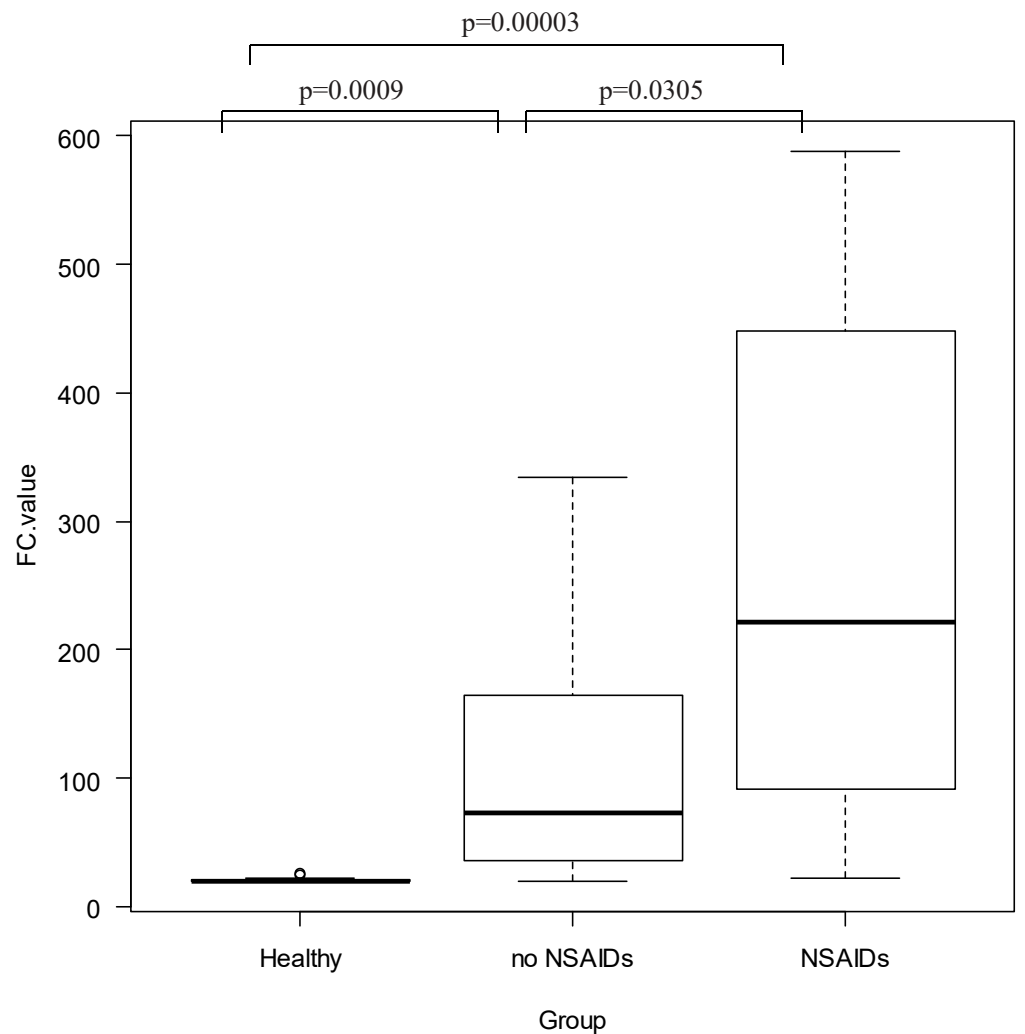

Fig. 1. Box-and-whisker plot of FC levels in study groups (bar $=$ median, box $=I Q R$, whiskers $=$ range, circles $=$ outliers $)$

\section{Correlations}

Then, we checked the correlation between indicators. We found the positive moderate-power correlations between the FC levels and the age (Spearman rank correlation coefficient $\rho=0.532$, $\mathrm{p}<0.000117)$, between the FC levels and the VAS score $(\rho=0.554, p=0.000117)$, between the FC levels and the time during which patients took NSAIDs $(\rho=0.479, p=0.00267)$ and the strong positive correlation between the FC levels and Lequesne index $(\rho=0.758, p<0.0001)$. We have not found the correlation between the FC levels and the Bristol scale or BMI $(\mathrm{p}>0.05)$.

\section{Qualitative comparisons}

We have checked the difference in frequencies of aspirin intake, statin intake, frequencies of detection of joint pain, stiffness, crepitations, and loose stools, and have not found the statistically significant difference in it $(\mathrm{p}>0.1)$. However, we found out that patients who took NSAIDs used proton pump inhibitors (PPI) significantly more frequently than healthy patients $(\mathrm{p}=0.03)$; there was no difference between OA groups in PPI intake or between those do not taking NSAIDs and healthy control $(\mathrm{p}>0.1)$. The OA groups were comparable by the frequency of concomitant pathology of gastrointestinal tract and joints $(\mathrm{p}=0.39)$, and by the frequency of symptoms from the gastrointestinal tract $(\mathrm{p}=0.96)$.

Next step, we checked if there is a difference in the frequency of FC elevation in the groups. We divided the elevation of the FC level into three groups based on the degree of increase of 
its level. The reference values were $19.5-49.9 \mathrm{mg} / \mathrm{kg}$ for normal, $50-199.9 \mathrm{mg} / \mathrm{kg}$ for elevated, $200 \mathrm{mg} / \mathrm{kg}$ and above for high level. The results are shown in the Fig. 2.

We found that the high FC levels are more common in patients taking NSAIDs than in those who do not $(\mathrm{p}=0.035, \mathrm{OR}=5.91$ [95 \% CI 1.102-43.330]). The difference in other FC levels of OA groups was not significant, but both OA groups are very different from the healthy control $(p<0.001)$ and did not differ significantly among themselves $(p>0.05)$.

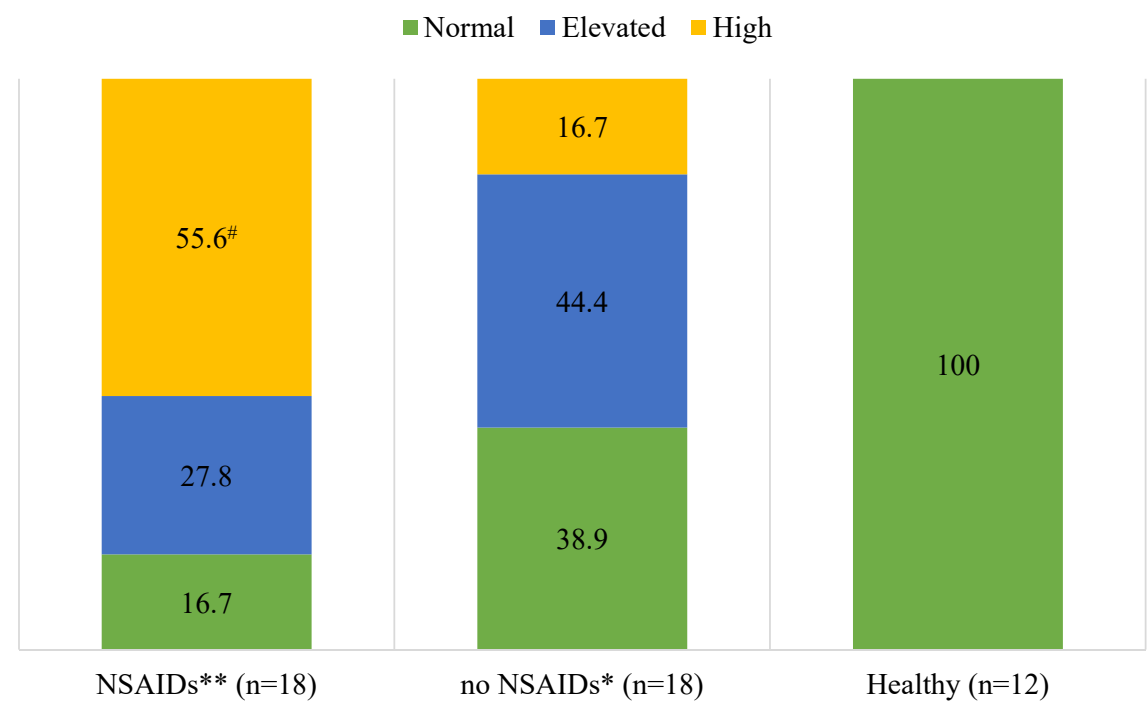

Fig. 2. The frequency of detection of different FC levels in the studied groups, \%:

* - the difference comparing to the healthy group is statistically significant $(p<0.01)$;

** - the difference comparing to the healthy group is statistically significant $(p<0.001)$;

\# - the difference comparing to the high FC level in no NSAIDs group is statistically significant $(\mathrm{p}=0.035)$

\section{Dependence on the NSAIDs type}

The last step, we divided all participants into three groups according to the selectivity of NSAIDs they take (or do not take). Because our patients took different NSAIDs of all types (high-selective, semi-selective and non-selective), we equated semi-selective drugs to selective ones and the group of those who do not take NSAIDs we combined with a healthy group. The results are shown on the Table $\mathbf{3}$ and Fig. $\mathbf{3}$.

Table 3

Medians of FC levels in groups depending on the type of NSAIDs used, $\mathrm{p}<0.001$

\begin{tabular}{ccc}
\hline Group & $\begin{array}{c}\text { Median (IQR), } \\
\mathbf{m g} / \mathbf{k g}\end{array}$ & $\begin{array}{c}\mathbf{9 5} \mathbf{\% C I}, \\
\mathbf{m g} / \mathbf{k g}\end{array}$ \\
\hline no NSAIDs $(\mathrm{n}=30)$ & $25.65(19.5-75.33)$ & $19.5-69.92$ \\
High-selective NSAIDs $(\mathrm{n}=2)$ & $98.53(91.56-105.5)$ & $91.56-105.5$ \\
Non-selective NSAIDs $(\mathrm{n}=16)$ & $264.1(89.72-464.67)$ & $103.66-448.61$
\end{tabular}

The difference in FC levels appeared to be significant only between non-selective NSAIDs and no NSAIDs group ( $\mathrm{p}=0.0003)$.

In the PPI intake, the difference was statistically significant only between non-selective and no NSAIDs group $(\mathrm{p}=0.038)$, and there was no difference between high-selective and non-selective groups $(\mathrm{p}=1)$. 


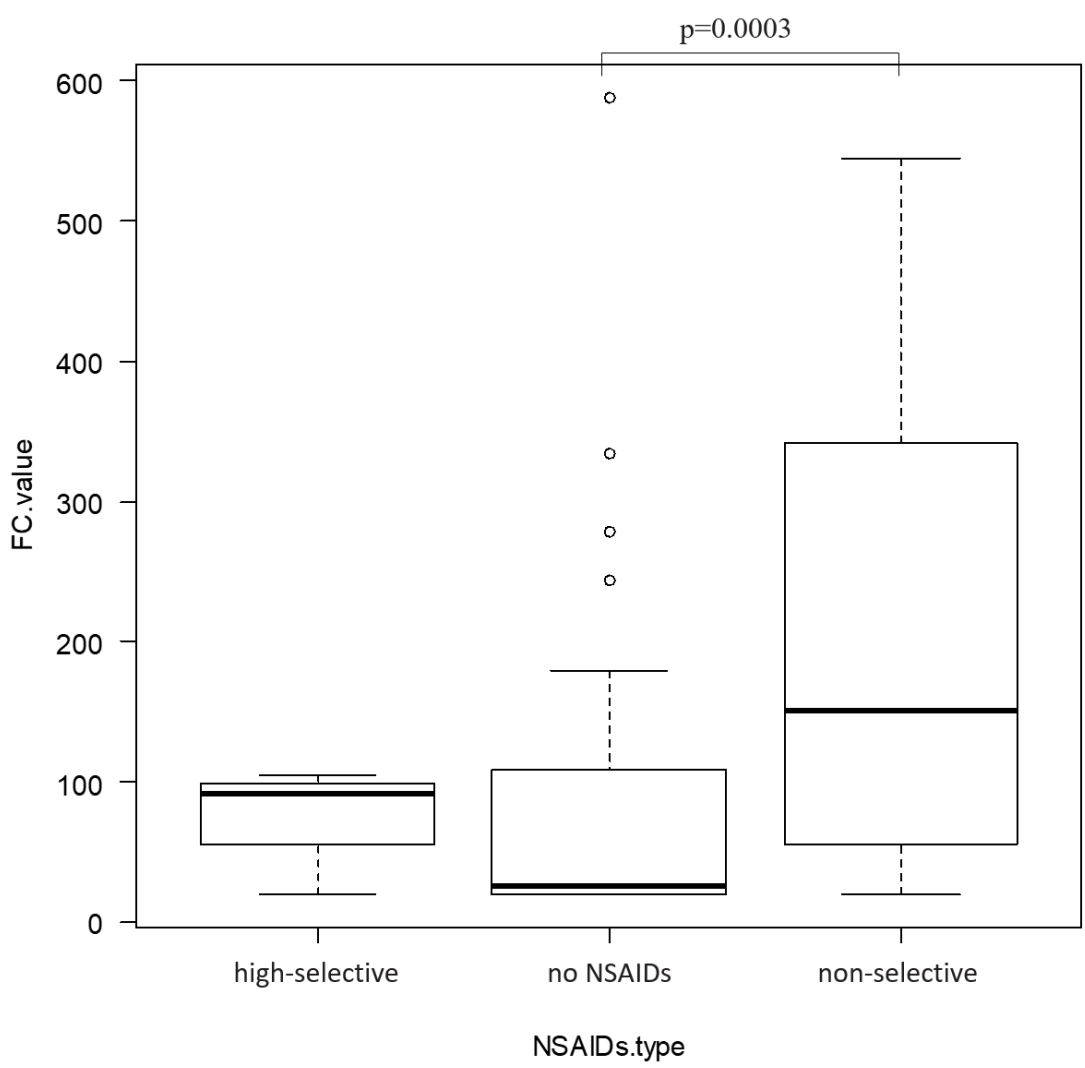

Fig. 3. Box-and-whisker plot FC levels in study groups according to the NSAIDs selectivity

\section{Discussion}

We found FC levels to be elevated the most in patients suffering from osteoarthritis and taking NSAIDs. OA patients who do not take NSAIDs have nearly three-times lower FC values, and healthy patients have normal FC values. Our results match the results of other investigators [8, 10], where participants, even healthy, who took NSAIDs, had similar FC values. The wide range in the FC values in NSAIDs group can be explained by different time the patients takes NSAIDs, as well as different NSAIDs types and individual characteristics of the separate patients. Need to notice that, in the study where healthy participants were given NSAIDs, some participants had their FC levels returned to normal even during 14-days NSAIDs intake [17]. The correlations we found seems to be mediated by NSAIDs intake.

We found the difference in the prevalence of PPI intake between OA groups. It can be the result of presence of other reasons in patients that did not take NSAIDs: some patients had conditions requiring PPI intake, such as GERD, another did not take NSAIDs for more than two weeks, but took PPI as a treatment for previously acquired NSAIDs-induced gastric ulcers.

Almost every third of OA patients in our investigation has high FC levels. According to literature data, high FC levels are associated with the presence of an undiagnosed gastrointestinal pathology. These patients should be investigated using endoscopic investigation methods. We have referred these patients to perform the colonoscopy.

We found the difference between FC levels in those who took non-selective NSAIDs and those who did not take any, but we did not manage to find any difference between FC levels in those who took high-selective NSAIDs and any other groups. On the other hand, this may be due to the little influence of high-selective NSAIDs on the intestine [18, 19], at least, with the short-term intake [20].

According to our results, there is no clear association between FC level and NSAIDs intake. As we can see from other studies, the FC level elevation due to the NSAIDs intake depends on the NSAIDs selectivity, and FC levels on the same equivalent dose of medication vary greatly [12]. This variation can be explained by other factors, including intestinal microbiota composition. Our other investigation highlights this problem [21]. 
Study limitations. This study was limited to patients suffering from the OA, and, thus, patients of the older age group. This study has a crossectional design, so it is limited in determining the causal relationships of the influence of the NSAIDs intake on FC elevation. We tried to check the influence of all possible factor on the FC levels, but some factors could be missed. The number of patients, especially those taking high-selective NSAIDs, was not large enough to find out all possible interconnections.

Prospects for further research. Further studies, including prospective ones, involving a more significant amount of subjects, especially those who take high-selective and half-selective NSAIDs, are needed to clarify the difference in the influence of NSAIDs of the different selectivity on the FC levels in studied category of patients.

\section{Conclusions}

The median FC level in our patients was 72.57 (IQR 20.55-221.57) $\mathrm{mg} / \mathrm{kg}, 95 \%$ CI 26,18-109.01.

I. OA patients had higher FC levels than the healthy group $(\mathrm{p}<0.001)$. OA patients who took NSAIDs had higher FC levels than those who did not take NSAIDs - $221.57 \mathrm{mg} / \mathrm{kg}$ (IQR 91.56$448.61)$ and $72.57 \mathrm{mg} / \mathrm{kg}(\mathrm{IQR} 35,26-164,79)$ respectively $(\mathrm{p}=0.03)$. The healthy participants had normal FC levels, and it differs from those who do not take NSAIDs $(\mathrm{p}<0.001)$. The FC levels have a sufficient positive correlation with the duration of NSAIDs intake, VAS score, and strong positive correlation with Lequesne index values.

II. When comparing FC levels in patients depending on the type of NSAIDs they take, we found that those who take non-selective NSAIDs have higher FC levels than those who do not take NSAIDs - 264.1 mg/kg (IQR 89.72-464.67) and $25.65 \mathrm{mg} / \mathrm{kg}$ (IQR 19.5-75.33) respectively $(\mathrm{p}=0.0003)$. The FC levels of those who take selective NSAIDs $-98.53 \mathrm{mg} / \mathrm{kg}(91.56-105.5)-$ did not differ significantly to FC levels in non-selective NSAIDs taker's group and no-NSAIDs group ( $\mathrm{p}>0.05)$.

Further research is needed in this area.

\section{Conflict of interests}

The authors declare that they have no conflicts of interest.

\section{Acknowledgements}

Authors kindly appreciate R-Biopharm AG, Darmstadt, Germany, for providing RIDASCREEN ${ }^{\circ}$ immunoassays for this work.

This study and article were inspired by the investigation of Z. Rendek et al. [17].

\section{References}

[1] Wallace, J. L. (2013). Polypharmacy of Osteoarthritis: The Perfect Intestinal Storm. Digestive Diseases and Sciences, 58 (11), 3088-3093. doi: http://doi.org/10.1007/s10620-013-2777-8

[2] Blackler, R. W., Gemici, B., Manko, A., Wallace, J. L. (2014). NSAID-gastroenteropathy: new aspects of pathogenesis and prevention. Current Opinion in Pharmacology, 19, 11-16. doi: http://doi.org/10.1016/j.coph.2014.05.008

[3] Balabanceva, A., Klyaritskaya, I., Tkach, S., Kuzenko, Y. (2014). SAT0117 Reduction of Nsaid-Induced Small Intestinal Damage in Rheumatoid Arthritis Patients Receiving Sulfasalazine. Annals of the Rheumatic Diseases, 73 (2), 633. doi: http:// doi.org/10.1136/annrheumdis-2014-eular.1671

[4] Gubska, O. J., Kuzminets, A. A. (2018). NSAID-enterotoxicity: focus on the problem. Modern Gastroenterology, 5, 50-59. doi: http://doi.org/10.30978/mg-2018-5-50

[5] Lim, Y. J., Yang, C.-H. (2012). Non-Steroidal Anti-Inflammatory Drug-Induced Enteropathy. Clinical Endoscopy, 45 (2), 138-144. doi: http://doi.org/10.5946/ce.2012.45.2.138

[6] Sostres, C., Gargallo, C. J., Lanas, A. (2013). Nonsteroidal anti-inflammatory drugs and upper and lower gastrointestinal mucosal damage. Arthritis Research \& Therapy, 15 (3), 3. doi: http://doi.org/10.1186/ar4175

[7] Costa, F., Mumolo, M. G., Bellini, M., Romano, M. R., Ceccarelli, L., Arpe, P. et. al. (2003). Role of faecal calprotectin as non-invasive marker of intestinal inflammation. Digestive and Liver Disease, 35 (9), 642-647. doi: http://doi.org/10.1016/ s1590-8658(03)00381-5 
[8] Maiden, L., Thjodleifsson, B., Theodors, A., Gonzalez, J., Bjarnason, I. (2005). A Quantitative Analysis of NSAID-Induced Small Bowel Pathology by Capsule Enteroscopy. Gastroenterology, 128 (5), 1172-1178. doi: http://doi.org/10.1053/j.gastro. 2005.03.020

[9] Thornjóðleifsson, B., Theodórs, A., Bjarnason, I. (2004). The effect of diclofenac on the small intestine studied by wireless endoscopy. Laeknabladid, 90 (10), 689-693. Available at: http://europepmc.org/abstract/MED/16819054

[10] Tibble, J. A., Sigthorsson, G., Foster, R., Scott, D., Fagerhol, M. K., Roseth, A., Bjarnason, I. (1999). High prevalence of NSAID enteropathy as shown by a simple faecal test. Gut, 45 (3), 362-366. doi: http://doi.org/10.1136/gut.45.3.362

[11] Meling, T. R., Aabakken, L., Røseth, A., Osnes, M. (1996). Faecal Calprotectin Shedding after Short-Term Treatment with Non-Steroidal Anti-Inflammatory Drugs. Scandinavian Journal of Gastroenterology, 31 (4), 339-344. doi: http://doi.org/ $10.3109 / 00365529609006407$

[12] Takeuchi, K., Smale, S., Premchand, P., Maiden, L., Sherwood, R., Thjodleifsson, B. et. al. (2006). Prevalence and Mechanism of Nonsteroidal Anti-Inflammatory Drug-Induced Clinical Relapse in Patients With Inflammatory Bowel Disease. Clinical Gastroenterology and Hepatology, 4 (2), 196-202. doi: http://doi.org/10.1016/s1542-3565(05)00980-8

[13] Lee, I. S., Cho, Y. K. (2015). Initial Steps to Prevent Nonsteroidal Anti-Inflammatory Drug- or Aspirin-Induced Enteropathy: Long-Term Outcome Data. Gut and Liver, 9 (6), 697. doi: http://doi.org/10.5009/gnl15287

[14] Carroccio, A., Iacono, G., Cottone, M., Di Prima, L., Cartabellotta, F., Cavataio, F. et. al. (2003). Diagnostic Accuracy of Fecal Calprotectin Assay in Distinguishing Organic Causes of Chronic Diarrhea from Irritable Bowel Syndrome: A Prospective Study in Adults and Children. Clinical Chemistry, 49 (6), 861-867. doi: http://doi.org/10.1373/49.6.861

[15] D’Angelo, F., Felley, C., Frossard, J. L. (2017). Calprotectin in Daily Practice: Where Do We Stand in 2017? Digestion, 95 (4), 293-301. doi: http://doi.org/10.1159/000476062

[16] Kanda, Y. (2012). Investigation of the freely available easy-to-use software "EZR" for medical statistics. Bone Marrow Transplantation, 48 (3), 452-458. doi: http://doi.org/10.1038/bmt.2012.244

[17] Rendek, Z., Falk, M., Grodzinsky, E., Wahlin, K., Kechagias, S., Svernlöv, R., Hjortswang, H. (2015). Effect of oral diclofenac intake on faecal calprotectin. Scandinavian Journal of Gastroenterology, 51 (1), 28-32. doi: http://doi.org/10.3109/00365521. 2015.1066421

[18] Wallace, J. L. (2011). NSAID gastropathy and enteropathy: distinct pathogenesis likely necessitates distinct prevention strategies. British Journal of Pharmacology, 165 (1), 67-74. doi: http://doi.org/10.1111/j.1476-5381.2011.01509.x

[19] Lim, Y. J., Chun, H. J. (2013). Recent Advances in NSAIDs-Induced Enteropathy Therapeutics: New Options, New Challenges. Gastroenterology Research and Practice, 2013, 1-7. doi: http://doi.org/10.1155/2013/761060

[20] Watanabe, T., Fujiwara, Y., Chan, F. K. L. (2019). Current knowledge on non-steroidal anti-inflammatory drug-induced small-bowel damage: a comprehensive review. Journal of Gastroenterology. doi: http://doi.org/10.1007/s00535-019-01657-8

[21] Gubska, O. Y., Kuzminetes, A. A., Hutsul, V. M., Lavrenchuk, I. O. (2019). Intestinal microbiome and osteoarthritis. GASTROENTEROLOGY, 53 (2), 132-137. doi: http://doi.org/10.22141/2308-2097.53.2.2019.168988

Received date 03.02.2020

Accepted date 17.03.2020

Published date 31.03.2020
(C) The Author(s) 2020

This is an open access article under the CC BY license (http://creativecommons.org/licenses/by/4.0). 